\title{
Constant Mean Curvature Spacelike Surfaces in Lorentzian Warped Products
}

\author{
Juan A. Aledo ${ }^{1}$ and Rafael M. Rubio ${ }^{2}$ \\ ${ }^{1}$ Departamento de Matemáticas, E.S.I. Informática, Universidad de Castilla-La Mancha, 02071 Albacete, Spain \\ ${ }^{2}$ Departamento de Matemáticas, Campus de Rabanales, Universidad de Córdoba, 14071 Córdoba, Spain
}

Correspondence should be addressed to Rafael M. Rubio; rmrubio@uco.es

Received 13 August 2015; Accepted 4 October 2015

Academic Editor: Jacopo Bellazzini

Copyright (C) 2015 J. A. Aledo and R. M. Rubio. This is an open access article distributed under the Creative Commons Attribution License, which permits unrestricted use, distribution, and reproduction in any medium, provided the original work is properly cited.

We characterize the spacelike slices of a Lorentzian warped product as the only constant mean curvature spacelike surfaces under suitable geometrical and physical assumptions. As a consequence of our study, we derive a Bernstein-type result which widely improves and extends the state-of-the-art results in this setting.

\section{Introduction}

Spacelike hypersurfaces in $(n \geq 3)$-dimensional Lorentzian spacetimes are geometrical objects of great physical and mathematical interest. Roughly speaking, each of them represents the physical space in a given instant of a time function. In electromagnetism, a spacelike hypersurface is an initial data set that univocally determines the future of the electromagnetic field which satisfies the Maxwell equations [1, Theorem 3.11.1], analogously, for the simple matter equations [1, Theorem 3.11.2]. In Causality Theory, the mere existence of a particular spacelike hypersurface implies that the spacetime obeys a certain causal property. Let us remark that the completeness of a spacelike hypersurface is required whenever we study its global properties, and, also, from a physical viewpoint, completeness implies that the whole physical space is taken into consideration. To know more details on the relevance of constant mean curvature spacelike hypersurfaces in General Relativity see [2].

From a mathematical point of view, the interest of spacelike hypersurfaces is motivated by their nice Bernstein-type properties. In fact, spacelike hypersurfaces of constant mean curvature $(\mathrm{CMC})$ in $(n \geq 3)$-dimensional spacetime are critical points of the area functional under a certain volume constraint [3]. When the ambient spacetime is the LorentzMinkowski spacetime $\mathbb{L}^{n}$, many results have been obtained from different viewpoints. For instance, as an application of the generalized maximum principle due to Omori-Yau $[4,5]$ and of the Calabi-Bernstein theorem, Aiyama [6] and Xin [7] (see also [8] for a first weaker version given by Palmer) obtained simultaneously and independently a characterization of spacelike hyperplanes as the only complete spacelike hypersurfaces with constant mean curvature (CMC) in the Lorentz-Minkowski spacetime whose hyperbolic image is bounded. As another application of the previous mentioned results, a characterization of the spacelike hyperplanes as the only complete CMC spacelike hypersurfaces in the LorentzMinkowski spacetime which lie between two parallel spacelike hyperplanes has been given by Aledo and Alías [9].

In this work, we will deal with spacelike surfaces but in a more general setting (some previous works in this scene, though with a completely different approach, are [10-12]; see Sections 4 and 5). Indeed, we will consider CMC spacelike surfaces in three-dimensional Lorentzian warped products, also called generalized Robertson-Walker spacetimes (see [13]). Note that in our study we make use (see Lemmas 1 and 7) of Lemmas 12 and 17 in [14], where the authors (with a completely different approach) study complete spacelike constant mean curvature hypersurfaces in warped products with parabolic fiber. Nonetheless, unlike our current study, all the principal results in [14] require the Timelike Convergence 
Condition and the hypothesis of boundedness of the hyperbolic angle between the normal vector field of the spacelike hypersurface and the timelike coordinate vector field of the warped product.

Finally, let us remark that although three-dimensional spacetimes may be considered unrealistic from a physical point of view, they have been deeply studied from a purely geometrical perspective. In fact, they can be used to light suitable extensions of geometrical properties to usual fourdimensional relativistic models.

The paper is organized as follows. In Section 2 we revise some notions regarding spacelike surfaces in a 3-dimensional Lorentzian space and introduce the notation to be used. We continue, in Section 3, undertaking some technical computations. Section 4 is devoted to present our main results. Thus, in Theorems 2 and 5 we characterize the CMC spacelike surfaces of a Lorentzian warped product as the only spacelike slices under suitable geometrical and physical assumptions. As a consequence of our study, in Section 5 we derive a Bernstein-type result (Theorem 8) which widely improves and extends the state-of-the-art results in this setting.

\section{Preliminaries}

Let $\left(F, g_{F}\right)$ be a connected Riemannian surface, $I$ an open interval in $\mathbb{R}$, and $f$ a positive smooth function defined on $I$. Then, the product manifold $I \times F$ endowed with the Lorentzian metric

$$
\bar{g}=-\pi_{I}^{*}\left(d t^{2}\right)+f\left(\pi_{I}\right)^{2} \pi_{F}^{*}\left(g_{F}\right),
$$

where $\pi_{I}$ and $\pi_{F}$ denote the projections onto $I$ and $F$, respectively, is called a Lorentzian warped product with fiber $\left(F, g_{F}\right)$, base $\left(I,-d t^{2}\right)$, and warping function $f$. Along this paper we will represent this 3-dimensional Lorentzian manifold by $\bar{M}=I \times_{f} F$.

The coordinate vector field $\partial_{t}:=\partial / \partial t$ globally defined on $\bar{M}$ is (unitary) timelike, and so $\bar{M}$ is time-orientable. We will also consider on $\bar{M}$ the conformal closed timelike vector field $\xi:=f\left(\pi_{I}\right) \partial_{t}$. From the relationship between the Levi-Civita connections of $\bar{M}$ and those of the base and the fiber [15, Corollary 7.35], it follows that

$$
\bar{\nabla}_{X} \xi=f^{\prime}\left(\pi_{I}\right) X
$$

for any $X \in \mathfrak{X}(\bar{M})$, where $\bar{\nabla}$ is the Levi-Civita connection of the Lorentzian metric (1). Thus, $\xi$ is conformal with $\mathscr{L}_{\xi}\langle\rangle=$, $2 f^{\prime}\left(\pi_{I}\right)\langle$,$\rangle and its metrically equivalent 1$-form is closed.

Recall that a Lorentzian manifold obeys the Null Convergence Condition (NCC) if its Ricci tensor $\overline{\mathrm{Ric}}$ satisfies $\overline{\operatorname{Ric}}(X, X) \geq 0$, for all null vector $X \in \mathfrak{X}(\bar{M})$. In the case, when $\bar{M}=I \times_{f} F$ is a Lorentzian warped product with a 2dimensional fiber, it can be easily checked that $\bar{M}$ obeys the NCC if and only if

$$
\frac{K^{F}\left(\pi_{F}\right)}{f^{2}}-(\log f)^{\prime \prime} \geq 0,
$$

where $K^{F}$ stands for the Gaussian curvature of $\left(F, g_{F}\right)$ (see, e.g., [11]).
Throughout this paper we will assume that $\left(F, g_{F}\right)$ is a complete Riemannian surface with finite total curvature; that is, its Gaussian curvature $K^{F}$ satisfies that

$$
\int_{F} \max \left\{0,-K^{F}\right\} d A_{F}<+\infty,
$$

where $d A_{F}$ is the area element of $\left(F, g_{F}\right)$ and the integral above is defined with a compact exhaustion procedure.

A smooth immersion $\psi: S \rightarrow \bar{M}$ of a (connected) surface $S$ is said to be a spacelike surface if the induced metric via $\psi$ is a Riemannian metric $g$ on $S$.

Since $\bar{M}$ is time-orientable we can take, for each spacelike surface $\psi: S \rightarrow \bar{M}$, a unique unitary timelike vector field $N \in \mathfrak{X}^{\perp}(S)$ globally defined on $S$ with the same time orientation as $-\partial_{t}$, such that $\bar{g}\left(N, \partial_{t}\right)>0$. From the wrong-way Cauchy-Schwarz inequality (see, e.g., [15, Proposition 5.30]), we have $\bar{g}\left(N, \partial_{t}\right) \geq 1$, and the equality holds at a point $p \in S$ if and only if $N=-\partial_{t}$ at $p$. In fact, $\bar{g}\left(N, \partial_{t}\right)=\cosh \theta$, where $\theta$ is the hyperbolic angle, at each point, between the unit timelike vectors $-\partial_{t}$ and $N$.

We will denote by $A$ and $H:=-(1 / n) \operatorname{trace}(A)$ the shape operator and the mean curvature function associated with $N$. A spacelike surface with constant $H$ is called a constant mean curvature (CMC) spacelike surface.

In any Lorentzian warped product $\bar{M}$ there is a remarkable family of spacelike surfaces, namely, its spacelike slices $\left\{t_{0}\right\} \times F, t_{0} \in I$. Note that a spacelike surface in $\bar{M}$ is a (piece of) spacelike slice if and only if the function $\tau:=\pi_{I} \circ \psi$ is constant. Furthermore, a spacelike hypersurface in $\bar{M}$ is a (piece of) spacelike slice if and only if the hyperbolic angle $\theta$ vanishes identically.

\section{Set Up}

Let $\psi: S \rightarrow \bar{M}$ be a spacelike hypersurface in a Lorentzian warped product $\bar{M}=I \times_{f} F$. If we put $\partial_{t}^{\top}=\partial_{t}+\bar{g}\left(\partial_{t}, N\right) N$, the tangential part of $\partial_{t}$, on $S$, it is easy to check that the gradient of $\tau=\pi_{I} \circ \psi$ on $S$ is given by

$$
\nabla \tau=-\partial_{t}^{\top}
$$

Now, from the Gauss formula and using $\xi^{\top}=f(\tau) \partial_{t}^{\top}$ and (5), the Laplacian of $\tau$ gives

$$
\Delta \tau=-\frac{f^{\prime}(\tau)}{f(\tau)}\left\{2+|\nabla \tau|^{2}\right\}-2 H \bar{g}\left(N, \partial_{t}\right),
$$

where $f^{\prime}(\tau):=f^{\prime} \circ \tau$.

On the other hand, in [11, Formula 10] the Gaussian curvature $K$ of a spacelike surface in $\bar{M}$ is computed and can be written as

$$
\begin{aligned}
K= & \left(\frac{f^{\prime}(\tau)^{2}}{f(\tau)^{2}}-H^{2}\right) \\
& +\left\{\frac{K^{F}\left(\pi_{F}\right)}{f(\tau)^{2}}-(\log f)^{\prime \prime}(\tau)\right\}|\nabla \tau|^{2}+\frac{K^{F}\left(\pi_{F}\right)}{f(\tau)^{2}} \\
& +\left(\frac{1}{2} \operatorname{trace}\left(A^{2}\right)-H^{2}\right) .
\end{aligned}
$$


We will use the following result [14, Lemma 12].

Lemma 1. Let $\psi: S \rightarrow \bar{M}$ be a CMC complete spacelike surface in $\bar{M}=I \times{ }_{f} F$ whose warping function $f$ is such that $(\log f)^{\prime \prime} \leq 0$. If the Gaussian curvature of $S$ is bounded from below and $S$ is contained between two slices, then

$$
H=-\frac{f^{\prime}(\tau)}{f(\tau)}
$$

Finally, note that, given a complete spacelike surface $\psi$ : $S \rightarrow \bar{M}$, the projection $\phi:=\pi_{F} \circ \psi: S \rightarrow F$ is a covering map provided that sup $f(\tau)<+\infty$ [13]. In particular, if the fiber $F$ is simply connected then $\phi$ is a diffeomorphism. Then, the area elements $d A_{S}$ and $d A_{F}$ of $(S, g)$ and $\left(F, g_{F}\right)$, respectively, satisfy the relationship,

$$
\phi^{*}\left(d A_{F}\right)=\frac{\cosh \theta}{f(\tau)^{2}} d A_{S}
$$

see [16, equation 8$]$.

\section{Main Results}

Next, we will characterize the spacelike slices under suitable geometrical and physical assumptions.

Theorem 2. Let $\left(F, g_{F}\right)$ be a simply connected Riemannian surface with finite total curvature and whose Gaussian curvature is bounded from below. Assume that the Lorentzian warped product $\bar{M}=I \times_{f} F$ satisfies the NCC and that the warping function $f$ is such that $(\log f)^{\prime \prime} \leq 0$.

Let $\psi: S \rightarrow \bar{M}$ be a CMC complete spacelike surface in $\bar{M}=I \times{ }_{f} F$. If $S$ is contained between two slices, then $S$ is a spacelike slice.

Proof. Since $\bar{M}$ satisfies the NCC, from (7) we get by using (3) that the Gaussian curvature $K$ of $S$ is bounded from below when $K^{F}$ is bounded from below. To see that, note that $f^{\prime}(\tau)^{2} / f(\tau)^{2}$ is bounded because $S$ is contained between two slices and that, from the Cauchy-Schwarz inequality, it is $\left((1 / 2) \operatorname{trace}\left(A^{2}\right)-H^{2}\right) \geq 0$. Then, from Lemma 1 we get that the (constant) mean curvature of $S$ is given by (8).

With all of this, the Gaussian curvature of $S(7)$ satisfies that

$$
K \geq \frac{K^{F}\left(\pi_{F}\right)}{f(\tau)^{2}} .
$$
that

Now, since $F$ has finite total curvature we have, using (9),

$$
\begin{aligned}
& \int_{S} \max \{0,-K\} d A_{S} \\
& \quad \leq \int_{F} \frac{1}{\cosh \left(\theta \circ \phi^{-1}\right)} \max \left\{0,-K^{F}\left(\pi_{F} \circ \phi^{-1}\right)\right\} d A_{F} \\
& \quad<+\infty .
\end{aligned}
$$

Therefore, $(S, g)$ has finite total curvature and, in particular, $(S, g)$ is parabolic.

Let us consider the function $F$ on $I$ given by

$$
F(t):=\int_{t_{0}}^{t} f(s) d s, \quad t_{0} \in I .
$$

Using (5), (6), and (8), the Laplacian of $F(\tau)$ can be computed to obtain

$$
\Delta F(\tau)=2 H f(\tau)(1+\cosh \theta)
$$

which is signed because $H$ is constant. Since $F(\tau)$ is bounded (because $S$ is contained between two slices), it follows from the parabolicity of $(S, g)$ that $F(\tau)$ is constant. Then

$$
0=\nabla F(\tau)=f(\tau) \nabla \tau
$$

and consequently $\nabla \tau=-\partial_{t}^{\top}=0$.

If we put $N^{F}=N+\bar{g}\left(N, \partial_{t}\right) \partial_{t}$, we get from $\bar{g}(N, N)=$ $-1=\bar{g}\left(\partial_{t}, \partial_{t}\right)$ that

$$
0=\left|\partial_{t}^{\top}\right|^{2}=\left|N^{F}\right|^{2}=\sinh ^{2} \theta
$$

that is, the hyperbolic angle vanishes identically on $S$ and therefore $S$ is a spacelike slice.

Remark 3. As we commented in Section 2, we need $\sup f(\tau)<+\infty$ to assure that $\phi$ is a diffeomorphism. In Theorem 2 this inequality holds since $\tau$ is bounded.

Note that the previous theorem is a wide extension of [9, Theorem 1]. To see this, it is enough to take a suitable splitting of $\mathbb{L}^{3}$.

A Lorentzian warped product $\mathbb{R} \times{ }_{e^{t}} F$, where $F$ is an $n$ dimensional Riemannian manifold, is called a steady state spacetime (see [17] for more details). The following result constitutes a partial extension of [17, Theorem 8], when $n=2$.

Corollary 4. Let $\mathbb{R} \times_{e^{t}} F$ be a 3-dimensional type steady state spacetime whose fiber is a simply connected Riemannian surface with finite total curvature and whose Gaussian curvature is bounded from below. Then, the only complete CMC spacelike surfaces contained between two slices are the spacelike slices.

Alternatively, the assumption of NCC can be changed for the one of bounded hyperbolic angle as follows.

Theorem 5. Let $\left(F, g_{F}\right)$ be a simply connected Riemannian surface with finite total curvature and whose Gaussian curvature is bounded from below. Assume that the warping function $f$ of the Lorentzian warped product $\bar{M}=I \times{ }_{f} F$ is such that $(\log f)^{\prime \prime} \leq 0$.

Let $\psi: S \rightarrow \bar{M}$ be a CMC complete spacelike surface in $\bar{M}=I \times{ }_{f} F$. If the hyperbolic angle of $\psi$ is bounded and $S$ is contained between two slices, then $S$ is a spacelike slice.

Proof. Reasoning as in the proof of Theorem 2, we get now that

$$
K \geq \frac{K^{F}\left(\pi_{F}\right)}{f(\tau)^{2}}\left(|\nabla \tau|^{2}+1\right)=\frac{K^{F}\left(\pi_{F}\right)}{f(\tau)^{2}} \cosh ^{2} \theta
$$


where we have also used (15). Then

$$
\begin{aligned}
& \int_{S} \max \{0,-K\} d A_{S} \\
& \quad \leq \int_{F} \cosh \left(\theta \circ \phi^{-1}\right) \max \left\{0,-K^{F}\left(\pi_{F} \circ \phi^{-1}\right)\right\} d A_{F} \\
& \quad<+\infty
\end{aligned}
$$

and the proof finishes as in Theorem 2.

Notice that the comment in Remark 3 is also valid for Theorem 5. On the other hand, it is worth pointing out that the boundedness of the surface and the one of the hyperbolic angle have no relation at all [12, Remark 5.3]. Hence, in Theorem 5, from the assumption of boundedness of the surface the boundedness of the hyperbolic angle cannot be derived and so this last condition must be required in order to conclude that $S$ is a spacelike surface.

The assumption of boundedness of the hyperbolic angle admits the following physical interpretation. Along $S$ there exist two families of instantaneous observers, $\mathscr{T}_{p}=-\partial_{t}(p)$, $p \in S$ (the sign minus depends on the time orientation chosen here), and the normal observers $N_{p}, p \in S$. The quantities $\cosh \theta(p)$ and $v(p):=(1 / \cosh \theta(p)) N_{p}^{F}$ are, respectively, the energy and the velocity that $\mathscr{T}_{p}$ measures for $N_{p}$, and we have $\|v\|=\tanh \theta$ on $S$. Therefore, the relative speed function satisfies $\|v\|<\lambda$ and so it does not approach the speed of light in vacuum on $S$.

In order to apply the previous results to more general cases we need an extra topological hypothesis.

Let us consider a GRW spacetime $M=I \times_{f} F$, whose fiber is a 2-dimensional complete Riemannian manifold. Recall that if the warping function is bounded on a complete spacelike surface $x: S \rightarrow M$, then

$$
\tilde{\pi}:=\pi_{F} \circ x: S \longrightarrow F
$$

is a covering map [13].

Now, let us take $p_{0} \in F$ and $\tilde{p}_{0} \in S$ such that $\phi\left(\tilde{p}_{0}\right)=p_{0}$. Denote by

$$
A=\frac{\pi_{1}\left(F, p_{0}\right)}{\widetilde{\pi}_{*}\left(\pi_{1}\left(S, \widetilde{p}_{0}\right)\right)}
$$

the set of all left cosets of $\tilde{\pi}_{*}\left(\pi_{1}\left(S, \widetilde{p}_{0}\right)\right)$ in $\pi_{1}\left(F, p_{0}\right)$. It is wellknown that

$$
\sharp\left(\tilde{\pi}^{-1}\left(p_{0}\right)\right)=\sharp(A) .
$$

Now, let us assume $\sharp(A)<\infty$. Thus, $S$ covers $\sharp(A)$ times the fiber. Moreover, taking into account the reasoning in Theorems 2 and 5, it is not difficult to see that $S$ also has finite total curvature under the same assumptions.

Remark 6. (a) Consider $F=\mathbb{S}^{1} \times \mathbb{R}$, endowed with its canonical product metric, and $f$, an arbitrary positive smooth function. Set $S=F$. For each positive integer $m$, let $x$ : $S \rightarrow I \times{ }_{f} F$ be the spacelike immersion given by $x\left(e^{i \theta}, s\right)=$ $\left(t_{0}, e^{i m \theta}, s\right)$. This example shows that there exist surfaces with arbitrary $\sharp(A)$. (b) However, we cannot force the fact that the fundamental group of the fiber is finite unless it is trivial. This is due to the fact that the fundamental group of any noncompact surface must be free (see, e.g., [18]).

We end this section pointing out that Theorems 2 and 5 extend and improve widely the conclusion given in [11, Corollary 6.11].

\section{Calabi-Bernstein-Type Problems}

Let $\left(F, g_{F}\right)$ be a (noncompact) complete Riemannian surface, $I \subset \mathbb{R}$ an open interval in $\mathbb{R}$, and $f$ a positive smooth function on $I$. For each $u \in C^{\infty}(F)$ such that $u(F) \subset I$ we can consider its graph $\Sigma_{u}:=\{(u(p), p): p \in F\}$ in the Lorentzian warped product $\bar{M}=I \times{ }_{f} F$. The graph of $u$ inherits a metric, represented on $F$ by $g_{u}:=-d u^{2}+f(u)^{2} g_{F}$, which is Riemannian if and only if $u$ satisfies $g_{F}(D u, D u)<$ $f(u)^{2}$ everywhere on $F$, where $D u$ denotes the gradient of $u$ in $\left(F, g_{F}\right)$. In this case, the graph is a spacelike surface in $\bar{M}$. Note that $\tau(u(p), p)=u(p)$ for any $p \in F$, and so, on the spacelike graph, $\tau$ and $u$ can be naturally identified.

When $\Sigma_{u}$ is spacelike, the unitary normal vector field on $\Sigma_{u}$ satisfying $\bar{g}\left(N, \partial_{t}\right)>0$ is

$$
N=-\frac{1}{f(u) \sqrt{f(u)^{2}-|D u|^{2}}}\left(f(u)^{2} \partial_{t}+D u\right),
$$

and the corresponding mean curvature function is

$$
\begin{aligned}
H(u)= & -\operatorname{div}\left(\frac{D u}{2 f(u) \sqrt{f(u)^{2}-|D u|^{2}}}\right) \\
& -\frac{f^{\prime}(u)}{2 \sqrt{f(u)^{2}-|D u|^{2}}}\left(2+\frac{|D u|^{2}}{f(u)^{2}}\right) .
\end{aligned}
$$

Our aim in this section is to study the entire solutions of the CMC spacelike surface equation

$$
\begin{aligned}
& \operatorname{div}\left(\frac{D u}{f(u) \sqrt{f(u)^{2}-|D u|^{2}}}\right) \\
& =-2 H-\frac{f^{\prime}(u)}{\sqrt{f(u)^{2}-|D u|^{2}}}\left(2+\frac{|D u|^{2}}{f(u)^{2}}\right), \\
& |D u|<\lambda f(u), \quad 0<\lambda<1,
\end{aligned}
$$

under suitable geometrical and analytical assumptions.

Note that the constraint (E.2) can be written as

$$
\cosh \theta<\frac{1}{\sqrt{1-\lambda^{2}}}
$$


where $\theta$ is the hyperbolic angle of $\Sigma_{u}$. Therefore, (E.2) implies that $\Sigma_{u}$ has bounded hyperbolic angle. Moreover, this constraint means that the differential equation (E.1) is, in fact, uniformly elliptic.

An important fact when we deal with entire graphs in this context is that, in general, the induced metric $g_{u}$ is not complete. In this sense, we will make use of the following result [14, Lemma 17].

Lemma 7. Let $\bar{M}=I \times_{f} F$ be a Lorentzian warped product whose fiber is a (noncompact) complete Riemannian surface. Consider a function $u \in C^{\infty}(F)$, with $\operatorname{Im}(u) \subseteq \mathrm{I}$, such that the entire graph $\Sigma_{u}=\{(u(p), p): p \in F\} \subset \bar{M}$ endowed with the metric $g_{u}=-d u^{2}+f(u)^{2} g_{F}$ is spacelike. If the hyperbolic angle of $\Sigma_{u}$ is bounded and $\inf f(u)>0$, then the graph $\left(\Sigma_{u}, g_{\Sigma_{u}}\right)$ is complete or, equivalently, the Riemannian surface $\left(F, g_{u}\right)$ is complete.

Now, from Theorem 5 and taking into account that every graph in a Lorentzian product is diffeomorphic to its fiber, we can state the following.

Theorem 8. Let $(F, g)$ be a simply connected Riemannian surface with finite total curvature and whose Gaussian curvature is bounded from below, and let $f: I \rightarrow \mathbb{R}$ be a smooth positive function such that $(\log f)^{\prime \prime} \leq 0$. Then, the only bounded solutions to the uniformly elliptic equation (E.1) + (E.2) are the constant functions.

Note that Theorem 8 thoroughly extends and improves [11, Theorem 7.1], as well as [12, Theorem 5.2].

\section{Conflict of Interests}

The authors declare that there is no conflict of interests regarding the publication of this paper.

\section{Acknowledgments}

The authors are grateful to the referee for his/her deep reading and making suggestions toward the improvement of this paper. The first author is partially supported by the Spanish Ministerio de Economía y Competitividad Grant with FEDER funds MTM2013-43970-P and by the Junta de Comunidades de Castilla-La Mancha Grant PEII-2014-001-A. The second author is partially supported by the Spanish MICINN Grant with FEDER funds MTM2013-47828-C2-1-P.

\section{References}

[1] R. K. Sachs and H. H. Wu, General Relativity for Mathematicians, vol. 48 of Graduate Texts in Mathematics, Springer-Verlag, New York, NY, USA, 1977.

[2] J. E. Marsden and F. J. Tipler, "Maximal hypersurfaces and foliations of constant mean curvature in General Relativity," Physics Reports, vol. 66, no. 3, pp. 109-139, 1980.

[3] A. Brasil Jr. and G. Colares, "On constant mean curvature spacelike hypersurfaces in Lorentz manifolds," Matematica Comtemporanea, vol. 17, pp. 99-136, 1999.
[4] H. Omori, "Isometric immersions of Riemannian manifolds," Journal of the Mathematical Society of Japan, vol. 19, no. 2, pp. 205-214, 1967.

[5] S. T. Yau, "Harmonic functions on complete Riemannian manifolds," Communications on Pure and Applied Mathematics, vol. 28, no. 2, pp. 201-228, 1975.

[6] R. Aiyama, "On the Gauss map of complete spacelike hypersurfaces of constant mean curvature in Minkowski space," Tsukuba Journal of Mathematics, vol. 16, pp. 353-361, 1992.

[7] Y. L. Xin, "On the Gauss image of a spacelike hypersurface with constant mean curvature in Minkowski space," Commentarii Mathematici Helvetici, vol. 66, no. 1, pp. 590-598, 1991.

[8] B. Palmer, "The Gauss map of a spacelike constant mean curvature hypersurface of Minkowski space," Commentarii Mathematici Helvetici, vol. 65, no. 1, pp. 52-57, 1990.

[9] J. A. Aledo and L. J. Alías, "On the curvatures of bounded complete spacelike hypersurfaces in the Lorentz-Minkowski space," Manuscripta Mathematica, vol. 101, no. 3, pp. 401-413, 2000.

[10] A. Romero and R. M. Rubio, "On the mean curvature of spacelike surfaces in certain three-dimensional Robertson-Walker spacetimes and Calabi-Bernstein's type problems," Annals of Global Analysis and Geometry, vol. 37, no. 1, pp. 21-31, 2009.

[11] M. Caballero, A. Romero, and R. M. Rubio, "Constant mean curvature spacelike surfaces in three-dimensional generalized Robertson-Walker spacetimes," Letters in Mathematical Physics, vol. 93, no. 1, pp. 85-105, 2010.

[12] M. Caballero, A. Romero, and R. M. Rubio, "New CalabiBernstein results for some elliptic nonlinear equations," Analysis and Applications, vol. 11, no. 1, Article ID 1350002, 13 pages, 2013.

[13] L. J. Alías, A. Romero, and M. Sánchez, "Uniqueness of complete spacelike hypersurfaces of constant mean curvature in Generalized Robertson-Walker spacetimes," General Relativity and Gravitation, vol. 27, no. 1, pp. 71-84, 1995.

[14] J. A. Aledo, R. M. Rubio, and A. Romero, "Constant mean curvature spacelike hypersurfaces in Lorentzian warped products and Calabi-Bernstein type problems," Nonlinear Analysis: Theory, Methods \& Applications, vol. 106, pp. 57-69, 2014.

[15] B. O'Neill, Semi-Riemannian Geometry With Applications to Relativity, Academic Press, 1983.

[16] J. Á. Aledo, A. Romero, and R. M. Rubio, "Upper and Lower bounds for the volume of a compact spacelike hypersurface in a generalized Robertson-Walker spacetime," International Journal of Geometric Methods in Modern Physics, vol. 11, no. 1, Article ID 1450006, 10 pages, 2014.

[17] A. L. Albujer and L. J. Alías, "Spacelike hypersurfaces with constant mean curvature in the steady state space," Proceedings of the American Mathematical Society, vol. 137, no. 2, pp. 711-721, 2009.

[18] A. Hatcher, Algebraic Topology, Cambridge University Press, Cambridge, UK, 2002. 


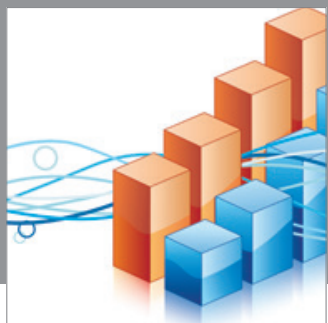

Advances in

Operations Research

mansans

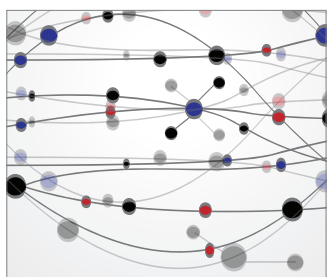

The Scientific World Journal
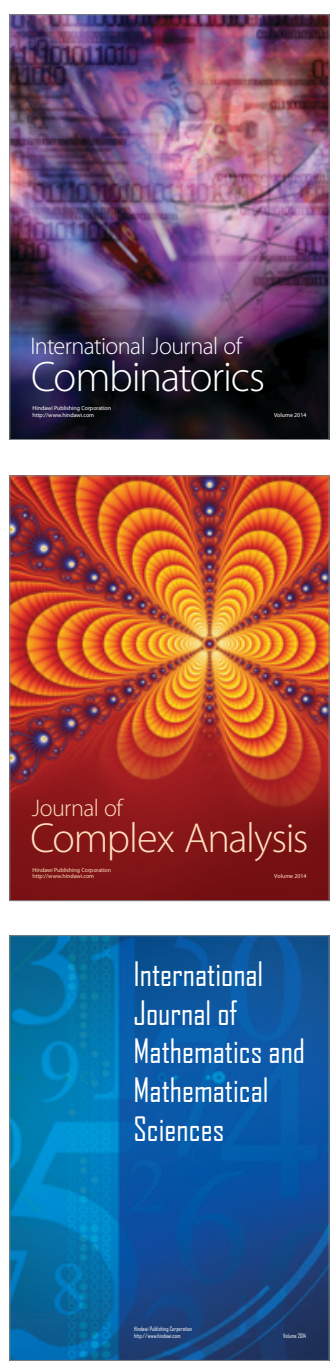
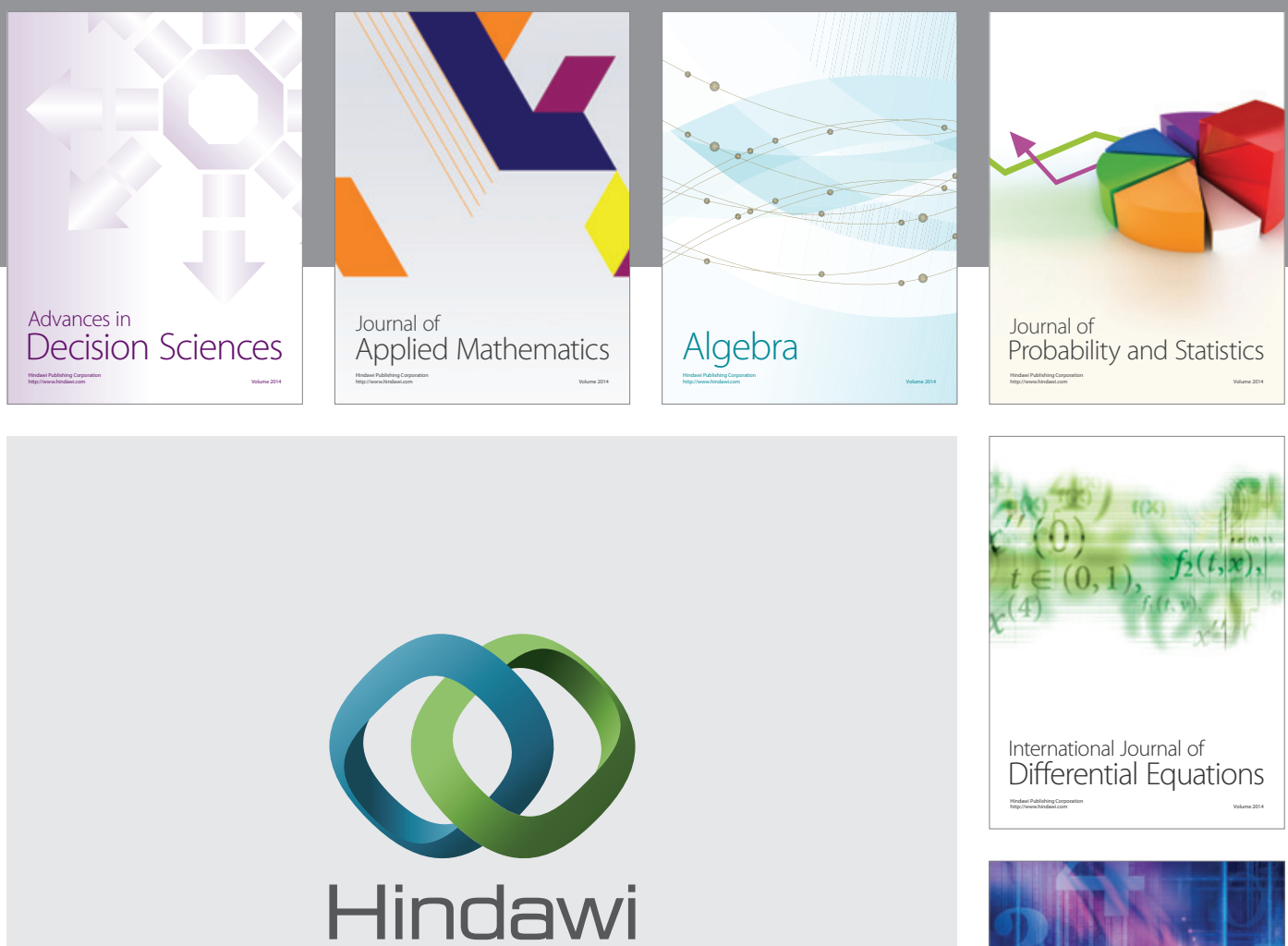

Submit your manuscripts at http://www.hindawi.com
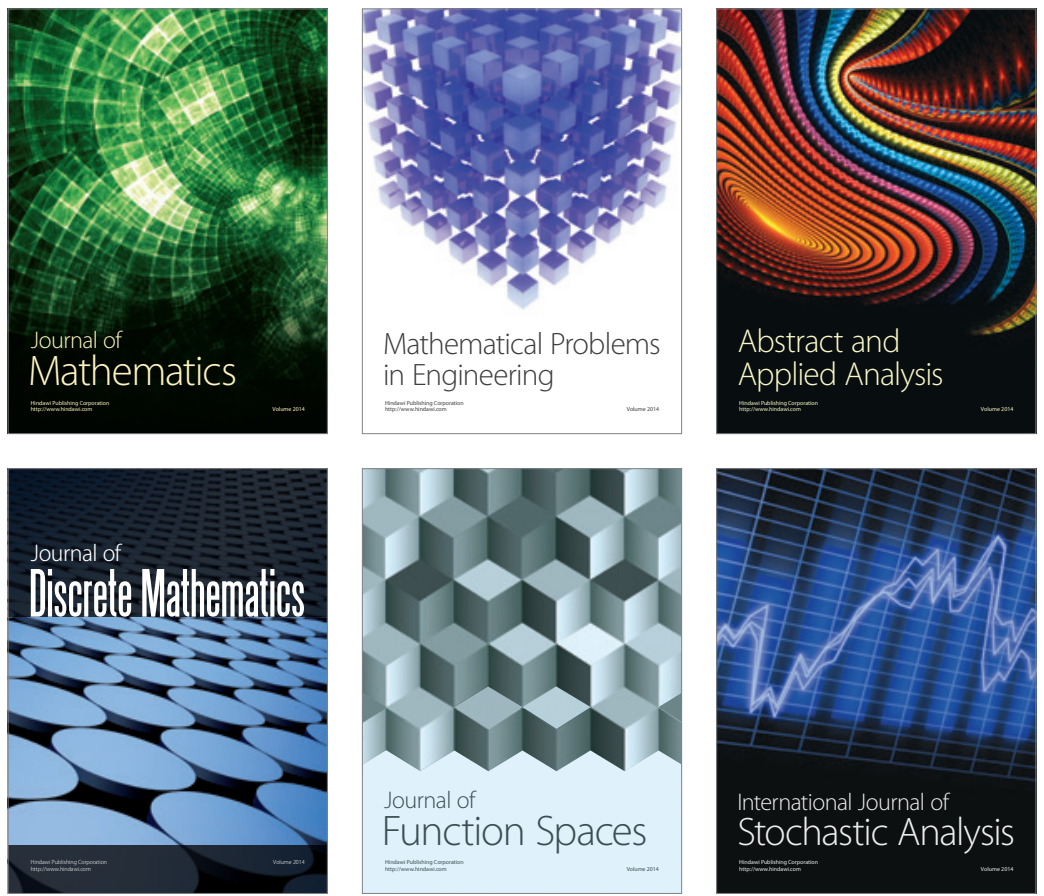

Journal of

Function Spaces

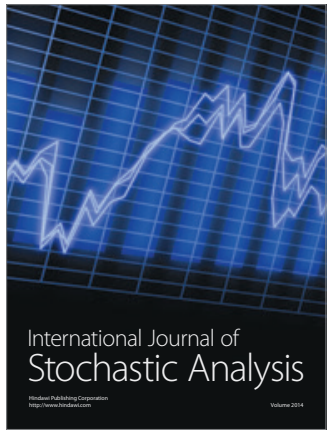

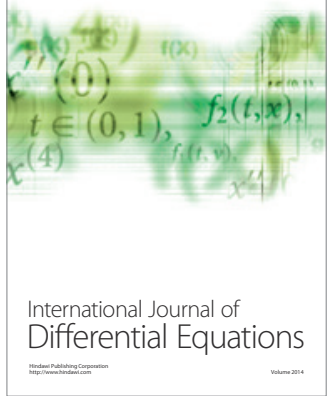
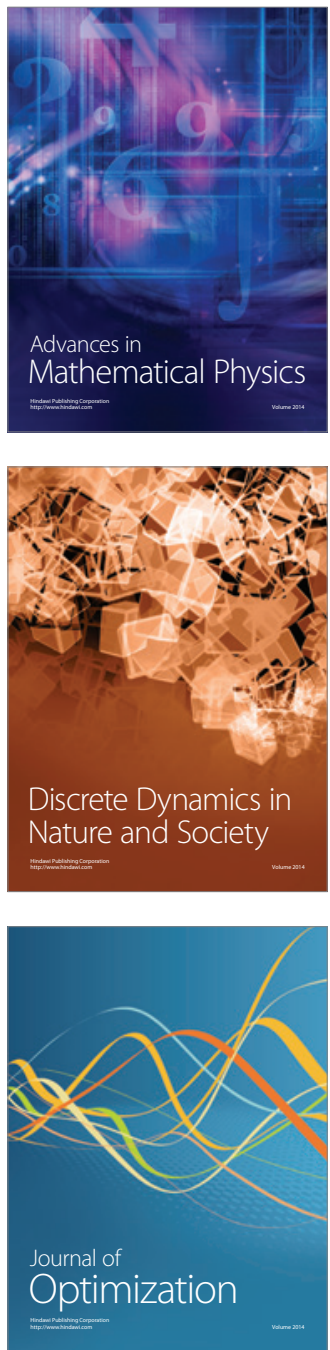